\title{
The journey from genetic predisposition to medication overuse headache to its acquisition as sequela of chronic migraine
}

\author{
Paolo Martelletti ${ }^{1,2}$
}

\begin{abstract}
Migraine remains one of the biggest clinical case to be solved among the non-communicable diseases, second to low back pain for disability caused as reported by the Global Burden of Disease Study 2016. Despite this, its genetics roots are still unknown. Its evolution in chronic forms hits $2-4 \%$ of the population and causes a form so far defined Medication Overuse Headache $(\mathrm{MOH})$, whose pathophysiological basis have not been explained by many dedicated studies. The Global Burden of Disease Study 2016 has not recognized MOH as independent entity, but as a sequela of Chronic Migraine. This concept, already reported in previous studies, has been confirmed by the efficacy of OnabotulinumtoxinA in Chronic Migraine independently from the presence of $\mathrm{MOH}$. The consistency of the current definitions of both Medication Overuse Headache and Chronic Migraine itself might be re-read on the basis of new evidences.
\end{abstract}

Keywords: Genetics, Migraine, Chronic migraine, Comorbidities, Medication overuse headache, Medication overuse, OnabotulinumtoxinA, Personalized medicine, Drug-drug-interactions

\section{Medication overuse headache: Chasing roots and Borders}

Medication Overuse Headache (MOH) is an underestimated social plague, that has always struggled to find research funds for epidemiological studies on large clinical records [1-3], probably due to non-scientific scotomata. The International Classification of Headache Disorders has interpreted $\mathrm{MOH}$ in an improper way through the years, and this has led to many clarifications and criticisms $[4,5]$. In fact, it is considered a headache secondary to the pharmacological abuse itself, without any consideration of the fact that $\mathrm{MOH}$ roots on Chronic Migraine (CM) [6]. Therefore, it must be considered as a complication of $\mathrm{CM}$ only. Rehabilitation from drug abuse, in whichever modality it is carried out, produces de facto a return to CM [7]. The same therapies applied today to $\mathrm{CM}$ are in fact efficacious with or without the

Correspondence: Paolo.Martelletti@uniroma1.it

${ }^{1}$ Department of Clinical and Molecular Medicine, Sapienza University, Rome, Italy

${ }^{2}$ Regional Referral Headache Centre, Sant'Andrea Hospital, Via di Grottarossa, 1035, 00189 Rome, Italy

Springer Open presence of $\mathrm{MOH}$, and an artificial distinction of a classification does not distinguish their efficacy [8-10].

Furthermore, we know that when migraineurs, whose genetic base remains yet undefined, despite the scientific efforts [11], develop CM plus Medication Overuse (MO) do not show univocal genetic patterns. Studies in this sector are at least jeopardized, and results correlate on various polymorphisms not always assonant, if we consider their action as drivers on possible physiopathological patterns [12-15]. Many refined functional neuroimaging studies applied to $\mathrm{MOH}$ undergo the same jeopardized criteria [16-18].

So, if we look for a generator of CM complication in $\mathrm{CM}$ plus $\mathrm{MO}$ we will have to look at the comorbidities spectrum, like depression, visceral pain, fibromyalgia, orofacial pain, temporo-mandibular disorders, myofascial trigger points that act on migraine incrementing its disability and an uncontrolled multiple need of analgesics [19]. This additional use disadvantages only migraine, since the patient with other osteoarticular pathologies who frequently uses analgesics and antimigraine drugs, if not migraneurs, will never develop any $\mathrm{MO}(\mathrm{H})$, ever. [19]. 


\section{Changing the classification of $\mathrm{MOH}$ as sequela of $\mathrm{CM}$} $\mathrm{MOH}$ doesn't have a genetic predisposition, then. Contradictory and varied studies did not lead to common denominator for a simple reason: $\mathrm{MOH}$ is not an entity independently classifiable, as the International Classification of Headache Disorders leads us to thin. It is a simple sequela, a complication of $\mathrm{CM}$, as we affirmed in $2011[4,5]$ and now validated in the recent Global Burden of Diseases 2016 (GBD2016) [19]. GBD2016 is to be considered today as the driving light on confirming, through epidemiology and disability parameters, that $\mathrm{MO}(\mathrm{H})$ is only a sequela of $\mathrm{CM}$ and must be summed in this clinical context adding a stronger value to migraine itself, now ranked second place, behind the low back pain only [20].

Therefore, the treatment for $\mathrm{CM}$, now based on the use of OnabotulinumtoxinA, should include the management of its sequela, making suddenly drop all the puzzles arisen after the registration studies, in which $2 / 3$ of enrolled CM patients had a co-diagnosis of $\mathrm{MOH}$. Such uncertainties have fallen with the following realworld studies with OnabotulinumtoxinA for $\mathrm{CM}$ where all treated patients were suffering from MO. The drug efficacy contextually reduced, and it couldn't be otherwise, headache days and consequently the excessive intake of medication.

The necessity of a definitive redefinition of this clinical situation of Chronic Migraine plus Medication Overuse [4] overwhelmingly arises. Figure 1 pieces together these clinical evidences.

We could shortly witness a new pharmacological era based on the use, in migraine treatment, of monoclonal antibodies against Calcitonin-Gene-Related-Peptide or its receptors, that if properly used in intercepting the evolution from high-frequency migraine towards its chronic phase will be able to reduce, in a decade, CM and its complication, MO [21].

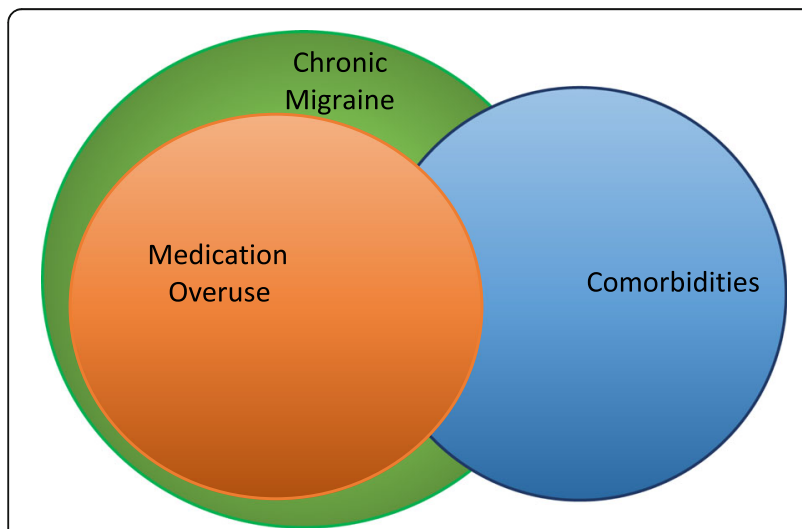

Fig. 1 Putative overlapping of Chronic Migraine, Medication Overuse and Migraine Comorbidities
Another matter recently opened on the solidness of the wall that supports the definition of chronic migraine, the 15 days per month of migraine, seems to waver on the basis of the brilliant study winner of the 2017 Greppi Award: the natural fluctuation above and below this barrier strongly challenge the conceptual modality of defining a form of migraine as chronic [22].

\section{Getting to the bottom of MO through personalized medicine}

We think today that MO originates from a numeric calculation and this causes overuse in a quasi-mysterious way. This cannot be all true. In fact, migraine experts prescribe acute and preventative therapies to mitigate pain and the symptomatology of existing comorbidities [23]. Real world setting migraine patients might also have other pathologies, not comorbid with migraine, that require additional therapies. This complex treatment may lead to many drug-drug interactions (DDIs), risking to develop not only Adverse Events (AEs) but also to reduce the clinical efficacy of the administered drug. This is particularly true in the case of migraine acute drugs leading to MO [24]. Genotype and phenotype also have an influence in the development of DDIs, therefore knowing them is crucial in choosing the best acute/preventative treatment and the appropriate drug interactions. The DrugBank, SuperCyp, PharmaGkb [23] are data banks containing all the potential genotypephenotype correlations in which it is possible to observe all the acknowledged DDIs and, knowing these, to recommend a tailored treatment avoiding metabolic clashes: in fact, more than $70 \%$ of prescribed drugs compete for the same metabolic pathway, the superfamily of cytochrome CYP450, and this represents the most common cause of drug reduction of efficacy and/or toxicity (Fig. 2). By avoiding this cross-competition we will be able to control this reduction of efficacy or drug toxicity and, therefore, the risk of MO. The Personalized Medicine model is particularly valid in migraine, where there is special need for multiple therapies due to the high number of comorbidities [25, 26]. With this clinicmolecular-biology-based approach, we will be able to obtain the better therapeutic performance, avoid DDIs, and practically combine acute, preventative and comorbidities treatments as well as additional sided therapies $[26,27]$. Personalized Medicine is the future for safe migraine treatment.

\section{Final re-reading}

Migraine is the second burdensome illness, if considered in terms of prevalence and disability. Its development in chronicity highlights important personal, organizational and health care problems. The 


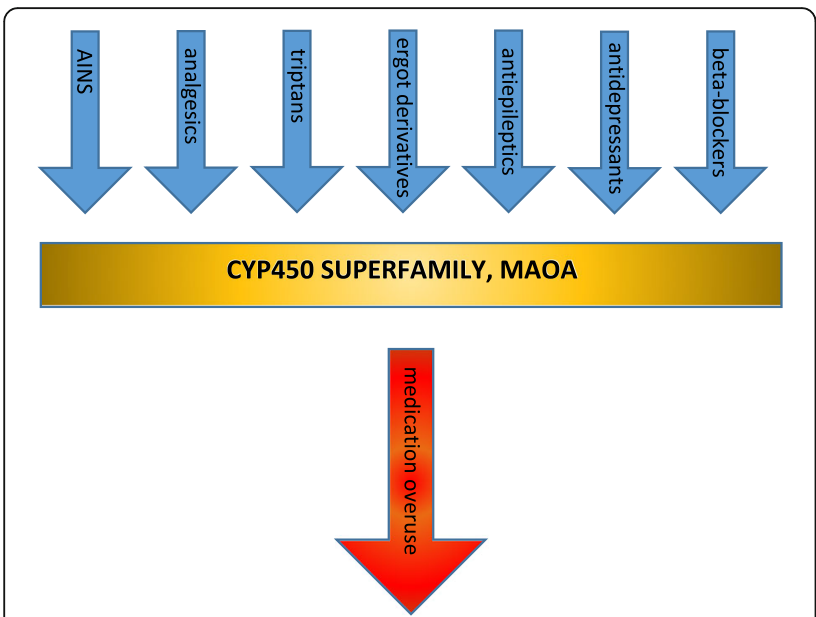

Fig. 2 Drug-drug interactions between preventative and acute migraine medications. The reported classes of drugs compete for the same metabolic pathway, and this represents the most common causes of drug reduction of efficacy and/or toxicity (for details please refer to ref. [23], specifically to tables 1 and 2), leading to medication overuse. Legenda AINS: anti-inflammatory non-steroidal drugs; CYP450: cytochromes P450 (accounting for the metabolism of more than $70 \%$ of prescribed drugs); MAOA: monoaminoxidase-A

distinction between $\mathrm{CM}$ and $\mathrm{MOH}$ has created cultural barriers in the management and rehabilitation. The natural fluctuations of headache days challenge the intrinsic definition of $\mathrm{CM}$ as we know it, and this clashes with the concept that migraine is a solidly progressive disorder. It is then necessary to bring $\mathrm{MOH}$ back to the simple definition of CM complication, where various genetic factors yet to be defined in large scales, multiple comorbidity factors and inadequate lifestyles might explain its development, and to re-read the $\mathrm{CM}$ core. The possibility in a near future to intercept migraine evolution from high frequency onward through the new pharmacological class of the monoclonal antibodies towards Calcitonin-Gene-Related-Peptide or its receptors might reduce its progression towards its fearsome complication, medication overuse.

\section{Financial Disclosure}

No financial support has been directly or indirectly received for the ideation, preparation and in writing this editorial.

\section{Competing interests}

The author declares that he has no competing interests related to the content of this Editorial.

Received: 21 December 2017 Accepted: 25 December 2017

Published online: 10 January 2018

\section{References}

1. Negro A, Curto M, Lionetto L, Guerzoni S, Pini LA, Martelletti P (2017) A critical evaluation on $\mathrm{MOH}$ current treatments. Curr Treat Options Neurol 19:32

2. Giamberadino MA, Mitsikostas DD, Martelletti P (2015) Update on medicationoveruse headache and its treatment. Curr Treat Options Neurol 17:368
3. Thorlund K, Sun-Edelstein C, Druyts E et al (2016) Risk of medication overuse headache across classes of treatments for acute migraine. $J$ Headache Pain 17:107

4. Negro A, Martelletti P (2011) Chronic migraine plus medication overuse headache: two entities or not? J Headache Pain 12:593-601

5. Farinelli I, Dionisi I, Martelletti P (2011) Rehabilitating chronic migraine complicated by medication overuse headaches: how can we prevent migraine relapse? Intern Emerg Med 6:23-28

6. Negro A, Curto M, Lionetto L, Giamberardino MA, Martelletti P (2016) Chronic migraine treatment: from OnabotulinumtoxinA onwards. Expert Rev Neurother 16:1217-1227

7. Cevoli S, Giannini G, Favoni V et al (2017) Treatment of withdrawal headache in patients with medication overuse headache: a pilot study. J Headache Pain 18:56

8. Davies B, Gaul C, Martelletti P, García-Moncó JC, Brown S (2017) Real-life use of onabotulinumtoxinA for symptom relief in patients with chronic migraine: REPOSE study methodology and baseline data. J Headache Pain 18:93

9. Negro A, Curto M, Lionetto L, Martelletti PA (2015) Two years open-label prospective study of OnabotulinumtoxinA $195 \mathrm{U}$ in medication overuse headache: a real-world experience. J Headache Pain 17:1

10. Negro A, Curto M, Lionetto L, Crialesi D, Martelletti P (2015) OnabotulinumtoxinA $155 \mathrm{U}$ in medication overuse headache: a two years prospective study. Spring 4:826

11. Kondratieva N, Azimova J, Skorobogatykh K et al (2016) Biomarkers of migraine: part 1 - genetic markers. J Neurol Sci 369:63-76

12. Cargnin S, Pautasso C, Viana M et al (2015) Association of RAMP1 rs7590387 with the risk of migraine transformation into medication overuse headache. Headache 55:658-668

13. Cargnin S, Viana M, Sances $G$ et al (2014) Combined effect of common gene variants on response to drug withdrawal therapy in medication overuse headache. Eur J Clin Pharmacol 70:1195-1202

14. Cargnin S, Viana M, Ghiotto N et al (2014) Functional polymorphisms in COMT and SLC6A4 genes influence the prognosis of patients with medication overuse headache after withdrawal therapy. Eur J Neurol 21:989-995

15. Gentile G, Borro M, Simmaco M, Missori S, Martelletti P. Gene polymorphisms involved in triptans pharmacokinetics and pharmacodynamics in migraine therapy. Exp Opin Drug Metab Toxicology 2011;7:39-47

16. Chen Z, Chen X, Liu M, Dong Z, Ma L, Altered YS (2017) Functional connectivity architecture of the brain in medication overuse headache using resting state fMRI. J Headache Pain 18:25

17. Chen Z, Chen X, Liu M, Liu S, Ma L, Texture YS (2017) Features of periaqueductal gray in the patients with medication-overuse headache. $J$ Headache Pain 18:14

18. Chen Z, Chen X, Liu M, Liu S, Ma L, Volume YS (2017) Gain of periaqueductal gray in medication-overuse headache. J Headache Pain 18:12

19. Giamberardino MA, Martelletti P (2017) In: Martelletti P (ed) Comorbitidies in headache disorders. Springer International Publishing, Switzerland, pp 1-217

20. GBD 2016 Disease and Injury Incidence and Prevalence Collaborators (2017) Global, regional, and national incidence, prevalence, and years lived with disability for 328 diseases and injuries for 195 countries, 1990-2016: a systematic analysis for the global burden of disease study 2016. Lancet 390:1211-1259

21. Martelletti P (2017) The application of CGRP( $r$ ) monoclonal antibodies in migraine spectrum: needs and priorities. BioDrugs 31:483-485

22. Serrano D, Lipton RB, Scher Al, Reed ML, Stewart WBF, Adams AM, Buse DC (2017) Fluctuations in episodic and chronic migraine status over the course of 1 year: implications for diagnosis, treatment and clinical trial design. $J$ Headache Pain 18:101

23. Lionetto L, Borro M, Curto M et al (2016) Choosing the safest acute therapy during chronic migraine prophylactic treatment: pharmacokinetic and pharmacodynamic consideration. Exp Opin Drug Metab. Toxicology 12:399-406

24. Lionetto L, Gentile G, Bellei E et al (2013) The omics in migraine. J Headache Pain 14:55

25. Borro M, Gentile G, Cipolloni L et al (2017) Personalized healthcare: the DiMA clinical model. Current Pharmaceutical Biothechnology 18:242-252

26. Simmaco M, Borro M, Missori S, Martelletti P (2009) Pharmacogenomics in migraine: catching biomarkers for a predictable disease control. Expert Rev Neurother 9:1267-1269

27. Bachall O (2015) Precision medicine. Nature 526:335 